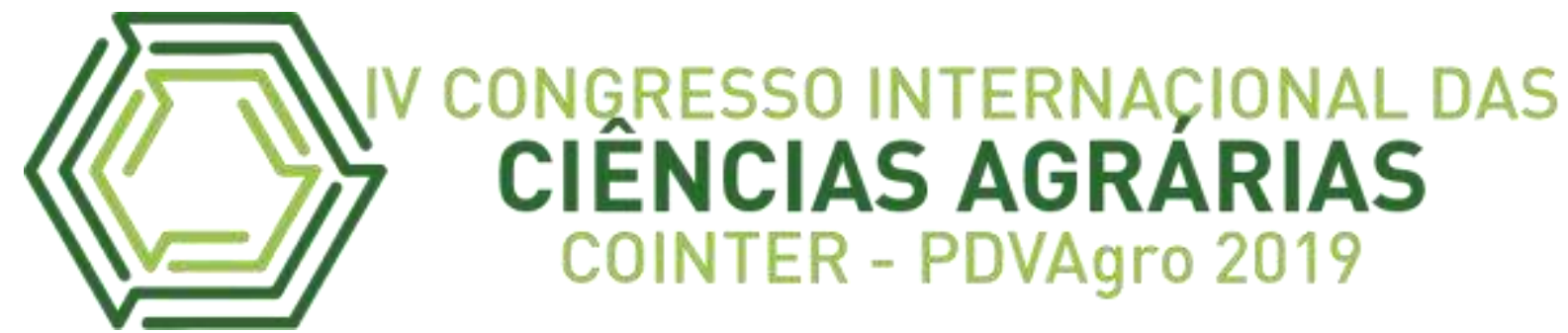

\title{
CRESCIMENTO DE Mytella charruana CULTIVADO EM MANGUEZAIS DE MACROMARÉ DA COSTA AMAZÔNICA, BRASIL
}

\section{CRESCIMIENTO DE Mytella charruana CULTIVADA EM LA COSTA DE MANGLAR DE MACRO MAREA AMAZÓNICA, BRASIL}

\section{GROWTH OF Mytella charruana CULTIVATED IN MACROTIDAL MANGROVES ON THE AMAZONIAN COAST, BRAZIL}

\author{
Apresentação: Comunicação Oral \\ Josinete Sampaio Monteles ${ }^{1}$; Maria Helena de Araújo Mendes ${ }^{2}$; Leuzanira Furtado Pereira ${ }^{3}$ \\ Paulo Protásio de Jesus ${ }^{4}$; Izabel Cristina da Silva Almeida Funo ${ }^{5}$
}

\section{DOI: $\underline{\text { https://doi.org/10.31692/2526-7701.IVCOINTERPDVAgro.2019.0022 }}$}

\begin{abstract}
Resumo
Ao longo da costa brasileira, muitas espécies de moluscos bivalves vêm sendo amplamente explorados como fonte de proteína e no incremento da renda familiar, dentre elas a Mytella charruana e Mytella guyanensis. Devido a produção, ainda não significativa desse bivalve, a pesquisa visou contribuir para o desenvolvimento da mitilicultura, oferecendo alternativa aos impactos ambientais da extração destes organismos, além de proporcionar maior produção alimentar à população. Para isso, os parâmetros produtivos da M. charruana e ambientais foram avaliados em cultivo experimental sob regime de macromarés no nordeste do Brasil. Os indivíduos de Mytella charruana utilizados no experimento foram adquiridos no local do estudo e o encordoamento realizado segundo método francês. Os coletores ficaram por 60 dias fixos ao sistema flutuante do tipo balsa e após esse período os sururus foram transferidos para o cultivo experimental. Foram confeccionadas 18 cordas de $50 \mathrm{~cm}$ de comprimento, com 420 sementes cada, cujo tamanho inicial médio era de $20,3 \mathrm{~mm}$. Os resultados apontaram que o crescimento do sururu $M$. charruana foi de cerca de $20,2 \mathrm{~mm}$ para altura da concha ao longo do experimento e que o crescimento mensal foi de $3,36 \mathrm{~mm}$. Já o ganho de peso foi de $3,9 \mathrm{~g}$ o que representou um ganho de peso mensal de $0,65 \mathrm{~g}$. Ao término do experimento constatou-se que o crescimento da altura da valva e peso total do sururu foi significativamente superior no período de estiagem. Uma vez que a análise de regressão não apontou influência significativa

\footnotetext{
${ }^{1}$ Graduanda de Licenciatura em Ciências Biológicas, Instituto Federal de Educação, Ciência e Tecnologia do Maranhão - Campus Monte Castelo, josimonteles@gmail.com

2 Discente do curso Técnico em Aquicultura, Instituto Federal de Educação, Ciência e Tecnologia do Maranhão Campus São Luís Maracanã, e-mail m.helena@acad.ifma.edu.br

${ }^{3}$ Graduando de Licenciatura em Ciências Agrárias, Instituto Federal de Educação, Ciência e Tecnologia do Maranhão - Campus São Luís Maracanã, paulo.protasio@acad.ifma.edu.br

${ }^{4}$ Graduanda de Licenciatura em Ciências Agrárias, Instituto Federal de Educação, Ciência e Tecnologia do Maranhão - Campus São Luís Maracanã, leuzafurtado@acad.ifma.edu.br

${ }^{5}$ Doutora em Recursos Pesqueiros e Aquicultura. Professora do Núcleo de Maricultura (NUMAR), Instituto Federal de Educação, Ciência e Tecnologia do Maranhão - Campus São Luís Maracanã, izabelfuno@ifma.edu.br
} 
das variáveis ambientais sobre crescimento, a hipótese para a diferença observada entre o período de estiagem e chuvoso pode estar relacionada à fauna incrustante e vágil associada. Em suma, o crescimento da $M$. charruana pode ser considerado satisfatório quando comparado com estudos pretéritos e contribuem para o desenvolvimento da atividade também em regiões sob regimes de grandes marés.

Palavras-Chave: Mytella charruana; Mexilhão; Macromarés; Parâmetros produtivos.

\title{
Resumen
}

A lo largo de la costa brasileña, muchas especies de moluscos bivalvos, como Mytella charruana y Mytella guyanensis, han sido ampliamente explotadas como fuente de proteínas y como complemento de sueldo familiar. La producción aún no significativa de este bivalvo, llevó a esta investigación, que posee como objetivo contribuir al desarrollo de la mitilicultura, de manera a ofrecer alternativas a los impactos ambientales de la extracción de estos organismos, $\mathrm{y}$, asimismo, proporcionar una mayor producción de alimentos a la población. Para ello, se evaluaron los parámetros ambientales y productivos de $M$. charruana en cultivos experimentales bajo régimen de macromareas en la región de Nordeste de Brasil. Los individuos de Mytella charruana utilizados en el experimento fueron adquiridos en el sitio de estudio y el encordado se realizó según el método francés. Los recolectores fueron fijados durante 60 días en sistema flotante (bateas) y después de este período los mejillones (llamados en esta región de sururu) fueron transferidos al cultivo experimental. Se hicieron 18 cuerdas de $50 \mathrm{~cm}$ de longitud, con 420 semillas a cada una, cuyo tamaño inicial promedio fue de $20.3 \mathrm{~mm}$. Los resultados revelaron que el crecimiento de mejillón (sururu) $M$. charruana fue de aproximadamente $20.2 \mathrm{~mm}$ para la altura de la concha durante todo el experimento y que el crecimiento mensual fue de $3.36 \mathrm{~mm}$. El aumento de peso fue de $3.9 \mathrm{~g}$, lo que representó un aumento de peso mensual de $0.65 \mathrm{~g}$. Al final del experimento, se observó que el crecimiento de la altura de valva y el peso total de mejillón (sururu) fue significativamente mayor durante el período de estiaje. Dado que el análisis de regresión no indicó una influencia significativa de las variables ambientales en el crecimiento, la hipótesis de la diferencia observada entre los períodos seco y lluvioso puede estar relacionada con incidencia de fauna incrustante y vágil asociada. En resumen, el crecimiento de $M$. charruana puede ser satisfactorio en comparación con estudios pretéritos y contribuye al desarrollo de la actividad también en regiones bajo regímenes de macromareas.

Palabras Clave: Mytella charruana; Mejillón; Macromareas; Parámetros productivos.

\begin{abstract}
Over the Brazilian coast, many species of bivalve molluscs have been widely explored as a protein source and increase of family income, among them Mytella charruana and Mytella guyanensis. With still not significant production, the research aimed to contribute to the development of the mussel farming, offer an alternative to the environmental impacts of extraction and provide greater food production for the population. For this, M. charruana productive parameters and environmental (oxygen, $\mathrm{pH}$, temperature, salinity, chlorophyll $a$ and suspended matter) were evaluated in experimental farming under macrotidal regime in northeastern Brazil. The individuals of M. charruana used in the experiment were recruited in site and the stranding performed according to the French method. The collectors were fixed for 60 days to the floating raft system type and thereafter mussels recruited were transferred to the experimental farming. 18 ropes with $50 \mathrm{~cm}$ in length, containing 420 seeds each, and having a
\end{abstract}


mean initial size of $20.3 \mathrm{~mm}$ were made. The results showed that $M$. charruana growth was about $20.2 \mathrm{~mm}$ for shell height during the experiment and that the monthly growth was $3.36 \mathrm{~mm}$. Already the weight gain was $3.9 \mathrm{~g}$ which represented a monthly weight gain of $0.65 \mathrm{~g}$. At the end of the experiment it was found that the growth of the valve height and total weight of mussels was significantly higher in the dry season. Since the regression analysis showed no significant influence of environmental variables on growth, the hypothesis for the observed difference between the dry and rainy periods may be related to the associated foul and fouling fauna. In short, $M$. charruana growth can be considered satisfactory when compared to previous studies and contribute to the development of activity also in regions under large tidal regimes.

Keywords: Mytella charruana. Mussel. Malacoculture. Macrotidal. Productive parameters.

\section{Introdução}

Em um processo histórico e silencioso as zonas costeiras, mares e oceanos sofreram com o processo da ocupação humana e as inúmeras formas de uso desses ambientes (MMA, 2010). O ecossistema mais visado e prejudicado, dentro deste cenário, foi o manguezal que associado a regiões estuarinas, se constitui um importante fornecedor de serviços ecológicos e recursos naturais para as comunidades costeiras (SILVA et al., 2000; ROCHA et al., 2008).

O complexo formado por estuário e manguezal abriga uma rica fauna de moluscos, peixes e crustáceos de relevante valor econômico (SILVA et al., 2000) que se constituem como principal fonte de renda e/ou complemento de renda familiar de milhares de pescadores artesanais como relata Monteles et al. (2009).

Entre os diversos grupos explorados no litoral brasileiro podemos citar os moluscos bivalves sarnambi ou berbigão (Anomalocardia brasiliana, Gmelin 1791), o sururu (Mytella charruana, Orbigny, 1846), a tarioba (Iphigenia brasiliensis, Lamark, 1818), a unha de velho (Tagelus plebeius, Lighffort, 1786) e a ostra (Crassostrea rhizophorae, Guilding, 1828) como os mais extraídos dos estuários e manguezais (SCHAEFFER-NOVELLI, 1989). Com a crescente demanda por alimentos em decorrência do aumento populacional, a malacocultura é apontada pela FAO (2009) como uma das alternativas para esta questão. Surge nesse cenário a mitilicultura (cultivo de mexilhões) como grande potencial para o desenvolvimento das comunidades de pescadores artesanais (ONODERA, 2012).

A mitilicultura, iniciada na Europa há aproximadamente 750 anos (ARANA, 1999) têm se destacado no cenário aquícola por seu baixo custo e facilidade de desenvolvimento (ONODERA, 2012). Dados da FAO (2009) demonstraram que a expansão desta atividade no ano de 2007 chegou a totalizar uma produção mundial de 1.630 .795 toneladas, com a China sendo responsável por 6.000 toneladas/ano desse total. 
Dados posteriores da FAO (2012) mostraram que a produção brasileira no ano de 2010 havia chegado a um total de 13.723 toneladas, sendo o Perna perna a principal espécie cultivada no Brasil (ONODERA, 2012) até aquele momento, 96\% da produção nacional. No entanto, segundo este autor, o Perna perna não era o único, havendo outros mitilídeos nativos importantes economicamente no Brasil como o Mytilus edulis platensis, no Rio Grande do Sul; Mytella guyanensis, com distribuição do Pará a Santa Catarina; e Mytella falcata (= Mytella charruana), encontrado nos estuários de toda costa brasileira (ONODERA, 2012). Porém, cabe ressaltar que ainda inviáveis do ponto de vista produtivo até aquele momento.

A exploração de sururu (espécies do gênero Mytella) em zonas entremarés, águas rasas costeiras, manguezais e estuários ocorrem principalmente por sua abundância, bem como pela facilidade de captura. No entanto, a exploração desordenada levou a exaustão de alguns bancos naturais e à preocupação com a sustentabilidade destes (MONTELES et al., 2009). Como alternativa para minimizar os impactos ambientais e proporcionar maior produção alimentar a população cada vez mais numerosa, torna-se imperativo o desenvolvimento de tecnologias que levem ao desenvolvimento de sistemas de cultivo, entre eles àqueles destinados a mitilicultura.

É visando contribuir com o desenvolvimento da mitilicultura no estado do Maranhão, que esta pesquisa se estruturou intencionando avaliar os parâmetros produtivos e ambientais em cultivo experimental do sururu $M$. charruana em manguezais de macromarés da costa amazônica (Raposa, Maranhão), Brasil, como forma de contribuir com o aprimoramento de metodologias de cultivo adequadas para a referida espécie, levando em consideração as características ambientais da região.

\section{Fundamentação Teórica}

A Costa de Manguezais de Macromaré da Amazônia (CMMA), estende-se da Baía de Marajó (Pará) até a Ponta de Tubarão, Baía de São José (Maranhão), perfazendo cerca de 650 $\mathrm{km}$ de litoral em linha reta (FILHO, 2005). A CMMA está sujeita a um regime de macromarés semidiurnas, com variações em torno de 4 m na Baía de Guajará, em Belém e 7,5 m na Baía de São Marcos (DHN, 2004). O clima é quente e úmido, com estação seca (julho a dezembro) e chuvosa (janeiro a maio) bem definidas, com precipitação média anual variando de 2500 a 3000 $\mathrm{mm}$ anuais e temperatura média em torno de $26^{\circ} \mathrm{C}$ (MORAES et al., 2005). A variação de maré ao longo do litoral maranhense é significativa podendo chegar a alguns pontos do litoral a altura de 9 metros, sendo que para a extensão do litoral do município de Raposa a amplitude de maré 
varia entre 4 e 7 metros (FSADU, 2010). De acordo com Funo (2016) é necessário a realização de pesquisas que avaliem os parâmetros produtivos e biológicos de moluscos bivalves nas regiões que sofrem influência das macromarés, com a perspectiva de contribuir com o aprimoramento de metodologias de cultivo adequadas para o desenvolvimento da malacocultura, levando em consideração às características ambientais da região.

A mitilicultura é o ramo da aquicultura que desenvolve o cultivo de mexilhões. Iniciada na Europa há aproximadamente 750 anos, é uma considerável fonte de alimento e renda para diversas populações litorâneas em vários países (ARANA, 1999). No Brasil, a principal espécie cultivada é o mexilhão Perna perna no Estado de Santa Catarina, responsável por $96 \%$ da produção nacional. No entanto, outros mitilídeos nativos são importantes economicamente no Brasil como o Mytilus edulis platensis, no Rio Grande do Sul; Mytella guyanensis, com distribuição do Pará a Santa Catarina; e Mytella charruana, encontrado nos estuários de toda costa brasileira. O gênero Mytella conhecido popularmente como sururu tem sido bastante explorado como fonte de alimento e renda em muitas regiões do Brasil com especial destaque aos gêneros Mytella guyanensis e $M$. falcata (=M. charruana), no entanto, ainda são inviáveis do ponto de vista produtivo. (ONODERA, 2012; MONTELES et al. 2017).

A espécie $M$. guyanensis tem sido estudada por diversos pesquisadores que avaliaram desde a biologia reprodutiva, recrutamento de sementes, e o crescimento até a taxa de sobrevivência em condições de cultivo (SIBAJA, 1986; BOLAÑOS, 1988; CRUZ; VILLALOBOS, 1993; LEONEL; SILVA; 1988; NISHIDA, 1988). Já para a M. falcata (=M. charruana) Pereira e Graça-Lopes (1995) avaliaram a captação e crescimento de sementes num experimento que buscava obtenção de sementes e engorda. Nos anos seguintes a taxa de crescimento dessa espécie foi avaliada por Costa e Nalesso (2002) e Sousa (2004). As pesquisas com a $M$. charruana têm se seguido ao longo dos anos em diferentes linhas. Para o litoral maranhense estudo realizado recentemente em manguezais de macromarés apresentaram resultados positivos no que diz respeito ao recrutamento de $M$. charruana em coletores artificias o que reforça o potencial de cultivo da espécie na região (MONTELES et al., 2017).

O litoral maranhense com seus $640 \mathrm{~km}$ de extensão apresenta potencial para o desenvolvimento da malacocultura (FSADU, 2010; FRANÇA et al., 2013). No entanto, o estado ainda não apresenta produção significativa no setor da malacocultura, o que demostra a necessidade de investimentos que viabilizem o processo produtivo de moluscos bivalves no litoral do estado do Maranhão. Assim, o investimento em pesquisa sobre o sururu e também o 
desenvolvimento de tecnologias de cultivo adequadas a essas espécies e as características ambientais do litoral maranhense poderão contribuir com o incremento da produção de ostra local. No entanto, estudos sobre os parâmetros produtivos do sururu Mytella charruana são escassos para essa região.

\section{Metodologia}

O estudo foi realizado em um estuário do município de Raposa - Maranhão, conhecido como Igarapé das Ostras, localizado sob as coordenadas geográficas $02^{\circ} 21^{\prime}$ a $02^{\circ} 32^{\prime}$ de latitude sul e $44^{\circ} 00^{\prime}$ a $44^{\circ} 12^{\prime}$ de longitude oeste, pertencente à Bacia Hidrográfica do Rio Paciência (Figura 1). A região estuarina de Raposa faz parte da Costa de Manguezais de Macromaré da Amazônia (CMMA), a qual se estende da Baía de Marajó (Pará) até a Ponta de Tubarão, Baía de São José (Maranhão), perfazendo cerca de $650 \mathrm{~km}$ de litoral em linha reta (FILHO, 2005).

As sementes de $M$. charruana utilizadas no experimento foram adquiridas no próprio local do estudo. Para isso, foram utilizados coletores artificiais confeccionados a partir de polietileno tereftalato conhecido popularmente como plástico do tipo PET. Para a construção do coletor, foram recortadas e sobrepostas placas de PET que serviram como substrato para que as sementes transportadas pelas correntes se fixassem. Uma vez instalados os coletores no ambiente estuarino, foram necessários 60 dias para que houvesse a fixação das sementes que, posteriormente, foram transferidas para o cultivo experimental. 
Figura 1: Igarapé das ostras, localizado em manguezais de macromarés da costa amazônica (Raposa, Maranhão),

Brasil

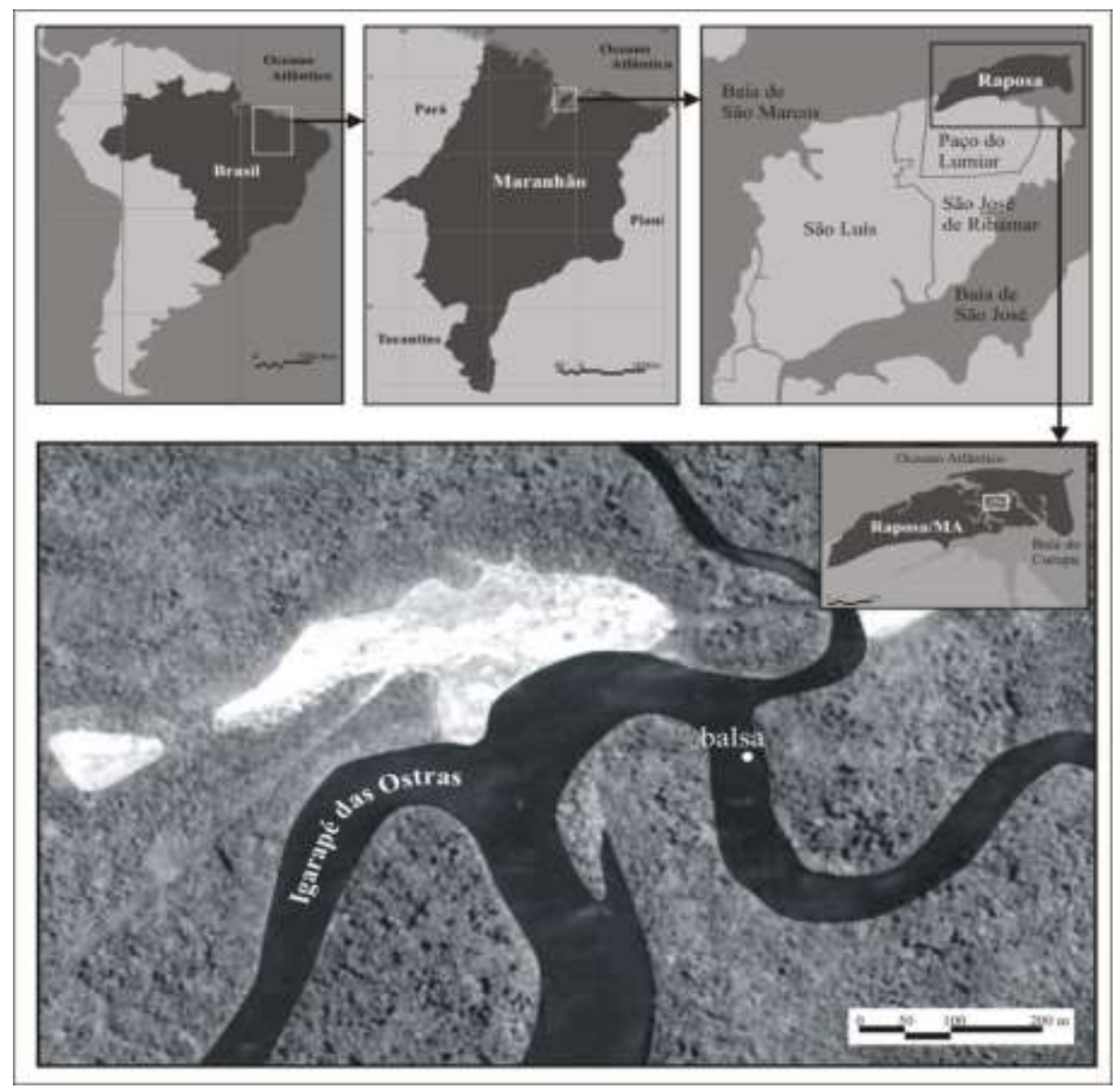

Fonte: Própria (2019)

No presente trabalho, foram confeccionadas 18 cordas com $50 \mathrm{~cm}$ de comprimento, e em cada corda foram acondicionadas 420 sementes de sururu, com tamanho inicial médio de 20,3 mm. O encordoamento foi realizado de acordo com o método francês, no qual os indivíduos são ensacados em um conjunto composto de duas redes tubulares de $60 \mathrm{~mm}$, formando dois sacos de rede, um dentro do outro. A primeira rede é de algodão e a externa é de poliamida. No interior das redes tubulares, passa um cabo central de nylon de 5 milímetros, esses materiais servem para garantir a sustentação das cordas no final do cultivo. Para facilitar o ensacamento do sururu (povoamento da corda) foi utilizado um cano PVC posteriormente retirado. As cordas foram fixadas no sistema de cultivo flutuante (balsa), onde permaneceram por 180 dias, 90 dias em cada período (estiagem e chuvoso). 
Para que fosse possível analisar o crescimento do sururu em função das variáveis ambientais, foram medidos mensalmente a temperatura e a salinidade da água do local do cultivo, usando um multiparâmetro (modelo YSI Multiprobe 556MPS. A avaliação da clorofila $a$ foi feita em laboratório a partir de amostras de água armazenadas e transportadas em caixas térmicas segundo o método descrito por Jeffrey e Humphrey (1975). Os dados de precipitação pluviométrica para o município de Raposa foram cedidos pelo Núcleo de Geoprocessamento da Universidade Estadual do Maranhão.

O procedimento de análise para determinar o crescimento mensal (altura e peso) dos sururus $M$. charruana em cultivo experimental foi realizado mensalmente. A cada mês, três cordas de cada tratamento foram retiradas da água. De cada corda, foram retiradas subamostras de 50 indivíduos medidos em relação à altura da concha $(\mathrm{mm})$, com auxílio de um paquímetro de precisão $0,1 \mathrm{~mm}$, e pesados para determinação do peso total (g) em uma balança digital com precisão de três decimais. A padronização das medidas de biometria das valvas dos sururus durante o experimento baseou-se no método proposto por Galtsoff (1964).

Durante o período experimental, foi feita uma análise qualitativa da fauna incrustante e vágil associada presentes nas estruturas de cultivo. O material foi fixado em formalina a $4 \%$ por 24 horas para posterior identificação em laboratório.

Ao término do período de amostragem, foram feitas análises estatísticas para avaliar os parâmetros de crescimento mensal da altura concha e do peso do sururu M. charruana. Para avaliar a significância entre as taxas de crescimento para período de estiagem e chuvoso foi utilizado o teste $t$ student com aplicação do teste de Monte Carlo. A normalidade dos dados foi verificada pelo teste de Shapiro-Wilk. Já para a correlação entre os parâmetros biológicos (crescimento da concha e do peso) e ambientais, foi empregada uma regressão linear visando identificar relação entre uma ou mais variáveis preditoras e a variável resposta. As análises estatísticas foram realizadas com auxílio dos programas estatísticos PAlaeontological STatistics (PAST) versão 3.25 e Microsoft Excel 2016.

\section{Resultados e Discussão}

Das cordas retiradas para amostragem, durante o período experimental, foi verificada boa fixação do sururu no cabo de nylon central, justificada pela uniformidade na distribuição dos mitilídeos ao longo da corda de cultivo (Figura 2). 
Figura 2: Cordas de sururu Mytella charruana manejadas durante o período experimental, em manguezais de macromarés da costa amazônica (Raposa, Maranhão), Brasil

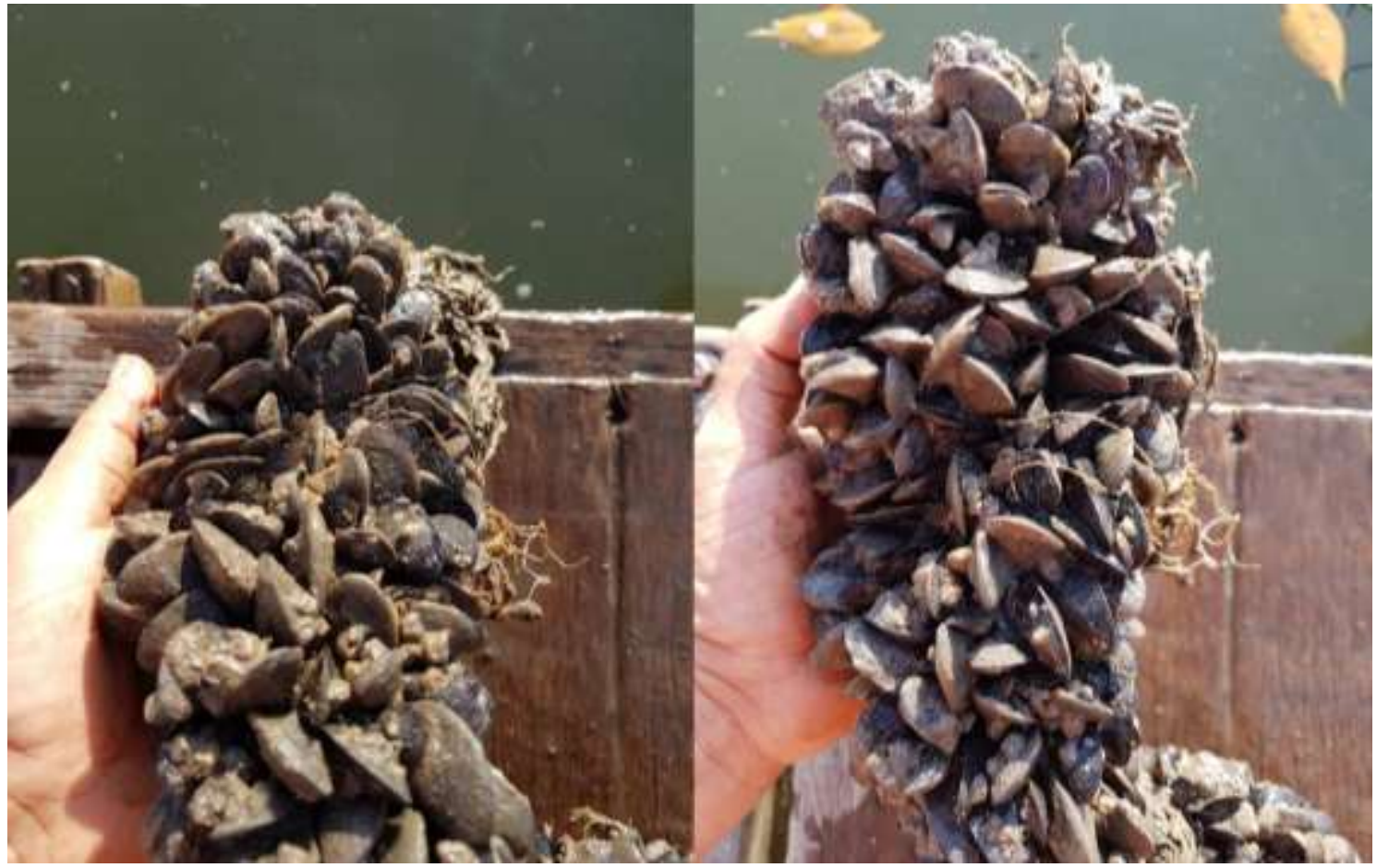

Fonte: Própria (2019)

A figura 3 apresenta a evolução dos dados de crescimento das conchas e peso total de Mytella charruana durante o período experimental. Os resultados obtidos nesta pesquisa apontam que o crescimento de Mytella charruana foi de $20,2 \mathrm{~mm}$ para a altura da valva $(3,36$ $\mathrm{mm} / \mathrm{mês})$ e de $3,9 \mathrm{~g}$ para o peso $(0,65 \mathrm{~g} / \mathrm{mês})$.

Contrapondo com os dados disponíveis na literatura, é possível perceber que os valores observados para o crescimento da $M$. charruana na região estuarina do estudo têm se mostrado satisfatórios. Isto porque, estudos pretéritos como o de Costa e Nalesso (2002), que avaliaram crescimento em $M$. falcata (=M. charruana) e $M$. guyanensis, obtiveram taxa de crescimento de apenas $1,8 \mathrm{~mm} / \mathrm{mês}$, valor abaixo do observado nesta pesquisa. Todavia, Câmara et al. (2018), avaliando o crescimento das espécies M. falcata (=M. charruana) e M. guyanensis no litoral maranhense, apontaram em seu estudo crescimento da valva de 3,0 e $5,7 \mathrm{~mm} / \mathrm{mês}$, e peso total de 0,6 e 0,9 g/mês, respectivamente. Os resultados do referido estudo, sobretudo da espécie M. charruana, são semelhantes aos obtidos na presente pesquisa, cujo crescimento médio mensal foi de 3,36 $\mathrm{mm}$ (altura da concha) e $0,65 \mathrm{~g}$ (peso total). Cabe ressaltar que a maior taxa de crescimento da $M$. guyanensis em relação a $M$. charruana deve-se a biologia da espécie que naturalmente tem altura de valvas maior. 
Figura 3: Média e desvio padrão dos dados de crescimento (altura da concha e peso total) de Mytella charruana cultivada em manguezais de macromarés da costa amazônica (Raposa, Maranhão), Brasil

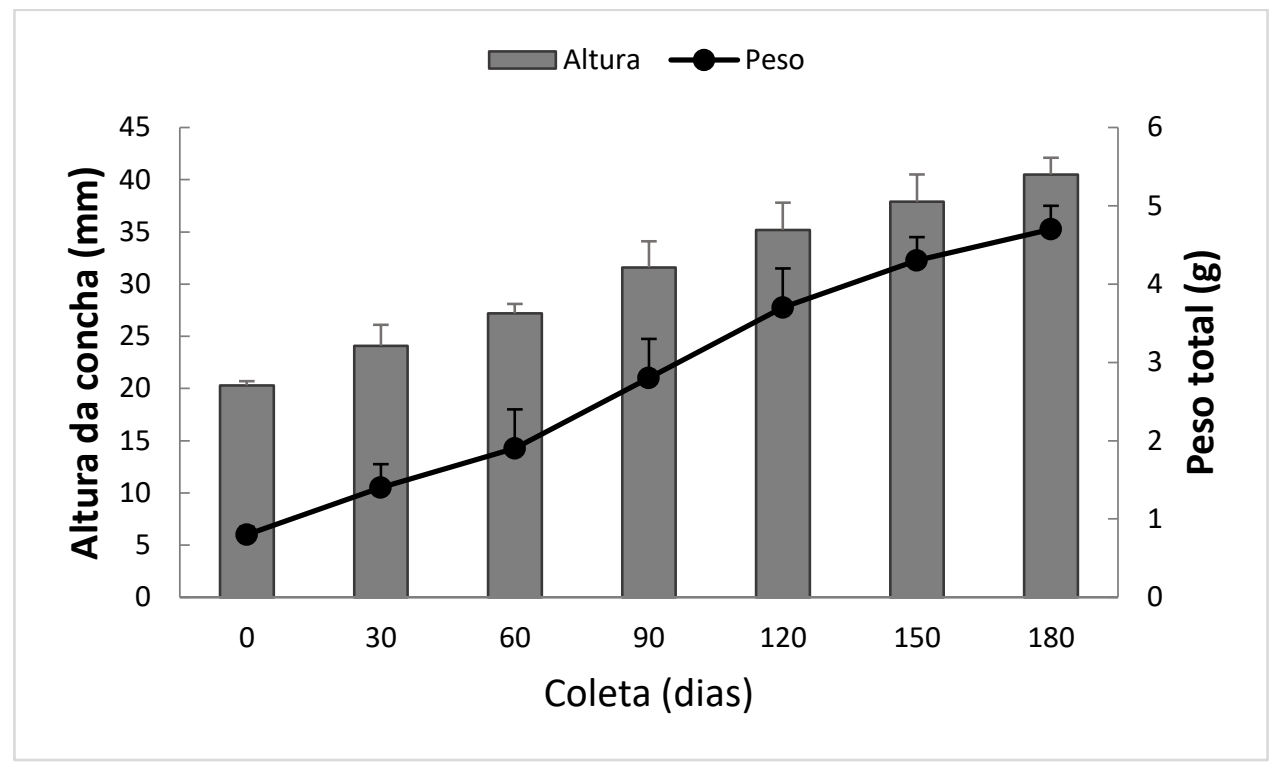

Fonte: Própria (2019)

De acordo com os resultados, foi verificado que durante o período do experimento, as variáveis ambientais não influenciaram significativamente sobre $\mathrm{o}$ crescimento da $M$. charruana (Tabela 1). Sendo a precipitação a variável que apresentou melhor correlação, embora não tenha sido significativa $(p>0,05)$. Na tabela 2, estão dispostos os dados ambientais e sua variação ao longo do período experimental.

Tabela 1: Relação entre variáveis ambientais e o crescimento de Mytella charruana cultivada em manguezais de macromarés da costa amazônica (Raposa, maranhão), Brasil

\begin{tabular}{lllllll}
\hline \multicolumn{8}{c}{ Coeficiente de Regressão e Estatística } & & & \\
\hline & & Coeficiente & Erro Padrão & $\mathrm{t}$ & $\mathrm{p}$ & $\mathrm{R}^{\wedge} 2$ \\
Altura $(\mathrm{mm})$ & Constante & 1,480 & 0,050 & 29,580 & 0,001 & \\
& Precipitação & 0,076 & 0,069 & 1,091 & 0,389 & 0,506 \\
& Salinidade & $-0,017$ & 0,176 & $-0,096$ & 0,932 & 0,215 \\
& Temperatura & $-0,028$ & 0,160 & $-0,172$ & 0,879 & 0,167 \\
& Clorofila & 0,035 & 0,280 & 0,126 & 0,911 & 0,213 \\
Peso $(\mathrm{g})$ & Constante & 0,378 & 0,130 & 2,909 & 0,101 & \\
& Precipitação & 0,209 & 0,180 & 1,158 & 0,367 & 0,491 \\
& Salinidade & $-0,021$ & 0,457 & $-0,045$ & 0,968 & 0,158 \\
& Temperatura & $-0,097$ & 0,415 & $-0,235$ & 0,836 & 0,149 \\
& Clorofila & 0,117 & 0,729 & 0,160 & 0,888 & 0,168 \\
\hline
\end{tabular}

Fonte: Própria (2019) 
Tabela 2: Média e desvio padrão das variáveis ambientais registradas no cultivo de Mytella charruana em manguezais de macromarés da costa amazônica (Raposa, Maranhão), Brasil

\begin{tabular}{ccccc}
\hline $\begin{array}{c}\text { Coleta } \\
\text { (dias) }\end{array}$ & Salinidade & $\begin{array}{c}\text { Temperatura } \\
\left(\mathbf{C}^{\mathbf{o}}\right)\end{array}$ & $\begin{array}{c}\text { Clorofila } \boldsymbol{a} \\
\left(\mathbf{m g . m}^{-\mathbf{3}}\right)\end{array}$ & $\begin{array}{c}\text { Precipitação } \\
(\mathbf{m m})\end{array}$ \\
\hline 0 & $31,2 \pm 2,8$ & $29,6 \pm 0,3$ & $4,4 \pm 1,1$ & 0,0 \\
30 & $32,0 \pm 4,6$ & $28,3 \pm 0,5$ & $4,2 \pm 2,6$ & 0,0 \\
60 & $32,3 \pm 3,7$ & $29,5 \pm 1,1$ & $4,8 \pm 3,3$ & 0,0 \\
90 & $33,0 \pm 5,8$ & $30,0 \pm 0,0$ & $5,0 \pm 2,8$ & 0,0 \\
120 & $32,0 \pm 0,7$ & $29,1 \pm 0,8$ & $4,3 \pm 0,4$ & 253,0 \\
150 & $30,0 \pm 2,1$ & $28,5 \pm 1,1$ & $3,9 \pm 1,0$ & 531,2 \\
180 & $29,2 \pm 0,5$ & $28,1 \pm 0,4$ & $3,5 \pm 0,2$ & 199,5 \\
\hline
\end{tabular}

Fonte: Própria (2019)

Avaliando o crescimento por meio da comparação de médias foi observado que em função da sazonalidade houve crescimento significativo da altura da concha no período de estiagem em comparação ao período chuvoso (4,0 e 2,7 mm, respectivamente) com valor de $\mathrm{p}=0,0001$ para o teste $\mathrm{t}$ student . $\mathrm{O}$ ganho de peso seguiu o mesmo padrão sazonal da altura da concha ( $\mathrm{p}=0,0001$; teste $t$ student $)$, com o período seco apresentando ganho significativamente maior de peso quando comparado ao chuvoso ( 0,8 e $0,5 \mathrm{~g}$, respectivamente).

As variáveis ambientais não influenciaram significativamente sobre o crescimento da M. charruana, apesar do maior crescimento das valvas e do peso total desses bivalves ter coincidido com o período em que foi determinada maior concentração de clorofila $a$ na água. Os valores da clorofila $a$, que representam a biomassa fitoplanctônica, variaram de 3,5 a 4,3 $\mathrm{mg} \cdot \mathrm{m}^{-3}$ no período chuvoso, enquanto no período de estiagem de 4,2 a 5,0 mg.m $\mathrm{m}^{-3}$ (Tabela 2).

A maior densidade de clorofila $a$ no período de menor precipitação pluviométrica corrobora com os resultados da pesquisa realizada por Azevedo et al. (2008), onde observaram maior concentração da clorofila no período de estiagem em estuários do Golfão Maranhense. Uma das explicações para o fato é a luz ser a principal variável para a produção primária. Uma vez que com as precipitações ocorre a diminuição da transparência da água, se espera que a redução da luz seja fator limitante para o fitoplâncton nos meses de maior pluviometria (PASSAVANTE; KOENING 1984; SASSI 1991, AZEVEDO et al., 2008).

Conforme pode ser observado na tabela 2 , a temperatura $\left(28\right.$ a $\left.30 \mathrm{C}^{\circ}\right)$ e salinidade $(29,2$ a 33) foram variáveis que pouco oscilaram ao longo do período de experimento, no entanto, segundo França et al., (2013) este padrão é característico dessa região. Funo et al (2019), para a mesma região, encontrou temperatura e salinidade superiores no período seco em relação ao chuvoso com máxima de salinidade de 40,5 e temperatura de $29,6^{\circ} \mathrm{C}$. Embora, não se 
constituam como fatores limitantes ao desenvolvimento do cultivo de $M$. charruana no município de Raposa-Maranhão, Yuan et al. (2010) relatam que a melhor faixa de sobrevivência da $M$. charruana está entre a faixa de salinidade de 2 e 23 sendo seus extremos fator de elevação da mortalidade.

No que diz respeito a organismos incrustantes e fauna vágil, observa-se que no período de estiagem o cultivo do sururu M. charruana foi infestado tanto por organismos incrustantes representados por cracas (Amphibalanus amphitrite e Amphibalanus improvisus) e por sementes de ostras (Ostrea equestris e Crassostrea spp.), quanto por fauna vágil, poliquetas do gênero Polydora sp. como ascídias. No período chuvoso, foi observado maior aglomeração do caramujo liso Stramonita haemastoma (Linnaeus, 1767) e sururus da espécie Mytella charruana se associando ao sistema de cultivo.

Estudo realizado no Golfão Maranhense por Feres (2010), visando avaliar a ameaça de organismos exóticos, identificou 24 espécies para a Ilha das Ostras, localizada no município de Raposa, Maranhão. Entre as espécies identificadas, o autor citou a ocorrência de alguns incrustantes encontrados no sistema de cultivo tais como Amphibalanus amphitrite e Amphibalanus improvisus, M. falcata (= Mytella charruana) e representantes do gênero Ostrea sp. De acordo com os estudos de Feres (2010), a espécie $M$. falcata (= M. charruana) apresenta maiores ocorrências no período de estiagem enquanto Amphibalanus amphitrite e Amphibalanus improvisus ocorrem o ano todo. A ocorrência desses organismos no sistema de cultivo torna-se um problema, uma vez que podem afetar o desenvolvimento da espécie cultivada por meio da competição.

No período de estiagem, um dos problemas relacionados ao cultivo foi a elevada ocorrência do poliqueta Polydora sp. Radashevsky e Migotto (2009) encontraram que representantes de Polydora hoplura levam ao desequilíbrio no desenvolvimento de moluscos, assim como na saúde destes. Em geral, estes anelídeos tornam-se prejudiciais aos cultivos porque conseguem adentrar o interior do molusco bivalve. O que levará a um aumento no gasto de energia para a produção de material calcário que recobrirá a perfuração feita pelo invasor. A energia gasta é desviada de funções vitais, como alimentação e reprodução o que acaba por afetar o desempenho produtivo em sistema de cultivo de moluscos. Por outro lado, no período chuvoso, houve a infestação de outros indivíduos da espécie $M$. charruana que se fixaram externamente à corda de cultivo. O elevado número de indivíduos ocupando o mesmo espaço 
e, portanto, competindo por alimento e espaço pode ter sido um dos fatores que elevou a mortalidade e reduziu o crescimento no período chuvoso.

Estudar especificamente a M. charruana e descobrir seu potencial para emprego em sistemas de cultivo proporcionará o desenvolvimento da atividade na região, que poderá produzir não só a $M$. guyanensis como também a M. charruana. Mas, sobretudo, proporcionará aprimoramento de metodologias de cultivo adequadas para a referida espécie mediante as condições ambientais específicas da região.

\section{Conclusão}

O trabalho, portanto, além de observar que o desempenho produtivo foi melhor no período de estiagem das chuvas e que não houve correlação significativa para o crescimento e a sobrevivência da espécie $M$. charruana com variáveis ambientais específicas, contribui com a inserção de técnicas produtivas (recrutamento e cultivo em corda suspensa) viáveis do ponto de vista econômico. O que permite que a atividade seja desenvolvida também por pescadores artesanais e não apenas grandes produtores.

Além do mais, os resultados sugerem que o crescimento e a sobrevivência significativamente maiores no período de estiagem estejam relacionados a menor interferência da fauna incrustante e vágil associada nesse período do cultivo.

\section{Agradecimentos}

Os autores agradecem ao Instituto Federal de Educação, Ciência e Tecnologia do Maranhão - IFMA, campus São Luís Maracanã pelo financiamento da pesquisa (Processo 23249.012106.2019-67) e à Fundação de Amparo à Pesquisa e Desenvolvimento Científico do Maranhão - FAPEMA, pela bolsa de iniciação científica concedida a Josinete Sampaio Monteles (Processo BIC-04330/18).

\section{Referências}

ARANA, L.V. Aquicultura e desenvolvimento sustentável: subsídios para a formulação de políticas públicas de desenvolvimento da aquicultura brasileira. EduUFSC: Florianópolis, 1999.

AZEVEDO, A.C.G. de; FEITOSA, F.A.N.; KOENING, M.L. Distribuição espacial e temporal da biomassa fitoplanctônica e variáveis ambientais no Golfão Maranhense, Brasil. Acta Botânica Brasílica, São Paulo, v. 22, n. 3, p. 870-877, 2008. 
BOLAÑOS, J. Estudio preliminar sobre el comportamento del mejillón Mytella guyanensis (Bivalvia: Mytilidade), em um parque fijo y uma estrutura flotante em la islã Chira. 117 p. Tesis de Licenciatura, Escuela de Biologia, Universidad de Costa Rica, 1988.

CÂMARA, A.M.de M.; MONTELES, J.S.; DE JESUS, P.P.; FERREIRA, V.F.; FUNO, I.C.S.A. Crescimento de Mytella charruana em manguezais da costa amazônica (Bequimão, Maranhão), Brasil. Apresentação oral - Anais da Feira Nacional do Camarão - FENACAM, 2018.

COSTA, K.G.; NALESSO, R.C. Cultivo experimental de Mytella falcata (Orbigny, 1846) e $M$. guyanensis (Lamarck, 1819), no estuário do Rio Piraquê-açu. (Aracruz-ES). Acta Limnologia Brasileiro, v.14, n.1, p.15-22, 2002.

CRUZ, R.A.; VILLALOBOS, C.R. Shell length at sexual maturity and spawning cycle of Mytella guyanensis (Bivalvia: Mytilidae) from Costa Rica. Revista de Biologia Tropical, v.41, p.89-92.1993.

DHN - DEPARTAMENTO DE HIDROGRAFIA E NAVEGAÇÃO. 2004. Tábuas de marés para 1994. Costa do Brasil e alguns portos estrangeiros. DHN, Rio de Janeiro, 1-6.

FAO - Food and Agriculture Organization. 2009 Global Aquaculture Production 1950-2007. Disponível em: http://www.fao.org/fishery/statistics/global-aquacultureproduction/query/en.Acesso em 12 de maio de 2009.

FAO. Food and Agriculture Organization. The State of World Fisheries and Aquaculture 2012. pp. 34-44, 2012.

FERES, Samir J.C. Organismos Exóticos: uma ameaça à sustentabilidade ambiental do Golfão Maranhense. 2010. Dissertação (Mestrado em Sustentabilidade de Ecossistemas) - Mestrado em Sustentabilidade de Ecossistemas, Universidade Federal do Maranhão, 2010.

FILHO, P.W.M.S. Costa de manguezais de macromaré da Amazônia: cenários morfológicos, mapeamento e quantificação de áreas usando dados de sensores remotos. Revista Brasileira de Geofísica. v. 23 n. 4, São Paulo, 2005.

FRANÇA, V.L. de; MONTELES, J.S.; ALMEIDA FUNO, I.C.S.; CASTRO, A.C.L. de. 2013. Seleção de áreas potenciais para o cultivo de Ostra nativa, Crassostrea spp. e Sururu, Mytella falcata, em Raposa, Maranhão. Arquivo Ciências do Mar, v.46, n.1, p.62-75.

FSADU. Fundação Sousândrade de Apoio. Plano Local de Desenvolvimento da Maricultura - PLDM's do Maranhão: Icatu, Humberto de Campos e Primeira Cruz. São Luís, 2010.

FUNO, I. C da S. A. Avaliação de parâmetros produtivos e biológicos da ostra nativa Crassostrea gasar (ADANSON, 1757) como subsídio ao desenvolvimento da ostreicultura em ambientes estuarinos do Estado do Maranhão. 2016. 122 f. Tese (Doutorado) - Universidade Federal Rural de Pernambuco. Recife, 2016. 
FUNO, I.C.S.A.; ANTONIO, I.G., MARINHO, Y.F.; MONTELES, J.S.; LOPES, R.G.P.S. \& GÁLVEZ, A.O. Recruitment of oyster in artificial collectors on the Amazon macrotidal mangrove coast. Ciencia Rural, Santa Maria, v. 49, n. 3, e20180482, 2019.

GALTSOFF, P.S. The American Oyster. Fishery Bulletin of the Fish and Wildlife Service, United States Departamento f the Interior, n.64, p. 480, 1964.

JEFFREY, S.W., HUMPHREY, G.F. New spectrophotometric equations for determining chlorophyll a, b, c and c in higher plants, algae and natural phytoplankton. - Biochem. Physiol. Pflanz. 167: 191-194, 1975.

LEONEL. R.M.V.; SILVA I.N. Estudo da sobrevivência e da capacidade de isolamento de Mytella guyanensis (Mollusca - Bivalvia) em diferentes salinidades. Revista Nordestina de Biologia, v.6, n.1, p. 35-41,1988.

MMA. Gerência de Biodiversidade Aquática e Recursos Pesqueiros. Panorama da conservação dos ecossistemas costeiros e marinhos no Brasil. Brasília: MMA/SBF/GBA, 2010. 148 p.

MONTELES, J.S., CASTRO, T.C.S. de, VIANA, D.C.P., CONCEIÇÃO, F.S., FRANÇA, V.L. de. FUNO, I.C.S. A. Percepção socioambiental das marisqueiras no município da Raposa-MA. Revista Brasileira de Engenharia de Pesca. v.4, n.2 p. 34-45, 2009.

MONTELES, J.S.; ASSUNÇÃO, J.M.; SILVA, E.O.; CÂMARA, A.M.de M.; FUNO, I.C.S.A. Recrutamento de semente de Mytella falcata em manguezais de macromaré no litoral maranhense. Apresentação oral - Anais da Feira Nacional do Camarão - FENACAM, 2017.

MORAES B. C.; COSTA, J. M. N.; COSTA, A. C. L. Variação espacial e temporal da precipitação no estado do Pará. Acta Amazônica, 35: 207-214, 2005.

NISHIDA, A. K. Alguns aspectos ecológicos de determinação da condição de Mytella guyanensis (Lamarck, 1819) (Mollusca - Bivalvia) da Ilha da restinga, estuário do Rio Paraíba do Norte, Paraíba, Brasil. Dissertação (Mestrado em Ciências Biológicas) Universidade Federal da Paraíba. Paraíba. 105 pp, 1988.

ONODERA, F.K. 2012. Mortalidade dos bivalves estuarinos, Mytella falcata e Mytella guyanensis, expostos a diferentes salinidades e temperaturas. Dissertação (Mestrado em Aquicultura e Pesca). Instituto de Pesca. APTA. 17-20pp.

PASSAVANTE, J.Z.O.; KOENING, M.L. 1984. Estudo ecológico da região de Itamaracá (Pernambuco - Brasil). XXVI Clorofila a e material em suspensão no estuário do rio Botafogo. Trabalhos Oceanográficos da Universidade Federal de Pernambuco v.18 p. 207-230,1984.

PEREIRA, O.M.; LOPES, R.G. 1995. Captação e crescimentos de sementes do sururu Mytella falcata (D'Orbigny, 1846) em coletores artificias, no Canal da Bertioga (23⒌'30" S, $45^{\circ} 13$ '42" W) Santos, São Paulo, Brasil. In: XIV Encontro Brasileiro de Malacologia. Anais. Porto Alegre. pp. 56. 
RADASHEVSKY, V.I.; MIGOTTO, A.E. Morphology and biology of a new Pseudopolydora species from Brazil. Journal of the Marine Biological Association of the United Kingdom, v.89, p.461-468, 2009.

ROCHA, M.S.P.; MOURÃO, J.S.; SOUTO, W.M.S.; BARBOZA, R.R.D.; ALVEZ, R.R.N. 2008. O uso dos recursos pesqueiros no estuário do rio Mamanguape, estado da Paraíba, Brasil. Interciência, v. 33, p.903-910, 2008.

SASSI, R. Phytoplankton and environmental factors in the Paraíba do Norte river estuary, southeastern Brazil: composition, distribution and quantitative remarks. Boletim do Instituto Oceanográfico v.39, p. 93-115, 1991.

SCHAEFFER-NOVELLI, Y. Perfil dos ecossistemas litorâneos brasileiros com especial ênfase sobre o ecossistema manguezal. Revista Instituto Oceanográfico, v.7, n.1, p.1-16, 1989.

SIBAJA, W.G.; VILLALOBOS, C. Crecimiento del mejillón chora Mytella guayanensis L (Bivalvía: Mytilidae) en el Golfo de Nicoya, Costa Rica. Revista de Biología Tropical, v. 34, pp. 231-36,1986.

SILVA, G.S. da; MELLO, R.L.S.; NASCIMENTO, A.E.; MESSIAS, A.S.; ARAÚJO, S.F.S. As atividades pesqueiras artesanais e a relação com a malacofauna no manguezal do rio Formoso, PE, Brasil. Trabalhos Oceanográficos da UFPE, Recife, v.28, n.2, p.195-207, 2000.

SOUSA, F.R. 2004. Avaliação da taxa de crescimento de Mytella falcata (Orbigny, 1846) em sistema de travesseiros, no povoado de Paquatiua, município de Alcântara-MA. Monografia apresentada ao curso de Licenciatura e Bacharel em Ciências Biológicas da UFMA, 42 p.

YUAN, W.; WALTERS, L. J.; SCHNEIDER, K. R.; HOFFMAN, E. A. Exploring the survival threshold: a study of salinity tolerance of the nonnative mussel Mytella charruana (Report), Journal of Shellfish Research, v.29, n.2, p.415-422. 2010. 